\title{
Sharp estimates \\ of the modified Hardy Littlewood maximal operator on the nonhomogeneous space via covering lemmas
}

\author{
Yoshihiro SaWano \\ (Received October 14, 2003; Revised February 2, 2004)
}

\begin{abstract}
In this paper we consider the modified maximal operator on the separable metric space. Define

$$
M_{k} f(x)=\sup _{r>0} \frac{1}{\mu(B(x, k r))} \int_{B(x, r)}|f(y)| d \mu(y)
$$

and

$$
M_{k, u c} f(x)=\sup _{x \in B(y, r)} \frac{1}{\mu(B(y, k r))} \int_{B(y, r)}|f(z)| d \mu(z)
$$

respectively. We investigate in what parameter $k$ the weak $(1,1)$-inequality holds for $M_{k}$ and $M_{k, u c}$ in general metric space and Euclidean space. The proofs are sharper than the method of Vitali's covering lemma. This attempt is partially done by Yutaka Terasawa [9] before. When we investigate $\mathbf{R}^{d}$, we prove a new covering lemma of $\mathbf{R}^{d}$. We also show that our condition on parameter $k$ is sharp. In connection with this we consider the dual inequality of Stein type and its applications.
\end{abstract}

Key words: maximal operator, covering lemma, non-homogeneous.

\section{Introduction}

The aim of this paper is to investigate maximal inequalities of the modified maximal operator associated to nondoubling measures. Let $(X, d)$ be a separable metric space endowed with a Radon measure $\mu$ such that all the balls are non-degenerate. We say that a ball $B$ with positive radius is non-degenerate if $\mu(B)>0$. For simplicity we assume that $\mu(B)<\infty$ for any ball $B$ with finite radius. In [8] the modified maximal operator is introduced as $\tilde{M} f(x)=\sup _{r>0} 1 / \mu(B(x, 3 r)) \int_{B(x, r)}|f(y)| d \mu(y)$, where $B(x, r)$ is an open ball with radius $r>0$ and center $x \in X$. They showed that $\mu(\{x \in X \mid \tilde{M} f(x)>\lambda\}) \leq 1 / \lambda \int_{X}|f(x)| d \mu(x)$.

Motivated by this, we define

2000 Mathematics Subject Classification : 42B25. 


$$
M_{k} f(x)=\sup _{r>0} \frac{1}{\mu(B(x, k r))} \int_{B(x, r)}|f(y)| d \mu(y) .
$$

Section 2 is devoted to the study of the weak- $(1,1)$ property of $M_{k}$. The strong- $(p, p)$ property and weak- $(1,1)$ property of $M_{k}$ still hold if $k \geq 2$.

Yutaka Terasawa [9] showed the following theorem.

Theorem 1.1 Let $X$ be a metric space (which is not necessarily separable). Let $\mu$ be a nondegenerate Radon measure such that $\mu(B(x, r))$ is continuous with the variable $r>0$ when $x \in X$ is fixed. Suppose that $k>$ 2 , then we have $M_{k}$ is weak- $(1,1)$ bounded and its weak- $(1,1)$ constant is less than or equal to 1 :

$$
\mu\left(\left\{x \in X \mid M_{k} f(x)>\lambda\right\}\right) \leq \frac{1}{\lambda} \int_{X}|f(x)| d \mu(x) .
$$

He proved the theorem using an outer measure method, which is different from the one we are going to use.

We shall prove the above theorem with $k=2$ using a new covering lemma.

Theorem 1.2 Let $(X, d)$ be a separable metric space. Let $\mu$ be a Radon measure such that $\mu(B(x, r))<\infty$. Then we have $M_{2}$ is weak- $(1,1)$ bounded. And the weak- $(1,1)$ constant is less than or equal to 1 :

$$
\mu\left(\left\{x \in X \mid M_{2} f(x)>\lambda\right\}\right) \leq \frac{1}{\lambda} \int_{X}|f(x)| d \mu(x) .
$$

Of course the strong- $(\infty, \infty)$ property is clear, by interpolation we only need to prove the weak- $(1,1)$ property to obtain the strong- $(p, p)$ property.

Yutaka Terasawa proposed the following question: In what parameter $k$, does $M_{k}$ satisfy the weak- $(1,1)$ estimate?

Motivated by this, we consider the following problem.

Question Does there exist a separable metric space such that $M_{k}$ is weak$(1,1)$ bounded only if $k \geq 2$ ?

Theorem 1.2 is sharp. We will show this sharpness in Section 2.3 by making an example whose property is summarized below.

Proposition 1.1 There exist a separable space $(X, d)$ and a measure $\mu$ such that $M_{k}$ is bounded if and only if $k \geq 2$. And the weak-(1,1) constant of $M_{2}$ is 1 on this space. 
Next we develop applications of this weak-type inequality and the covering lemma used to prove Theorem 1.2. First we derive the dual inequality by the method used in Theorem 1.2. Next using the duality inequality carefully, we derive the Fefferman-Stein type vector-valued inequality for the nonhomogeneous space. The result we will get is the following.

Theorem 1.3 If $1<p<\infty, 1<q \leq \infty$, then we have

$$
\left\|\left(\sum_{j \in \mathbf{Z}}\left(M_{l} f_{j}\right)^{q}\right)^{1 / q}\right\|_{p} \leq C_{p, q}\left\|\left(\sum_{j \in \mathbf{Z}}\left|f_{j}\right|^{q}\right)^{1 / q}\right\|_{p},
$$

if $l$ is large enough. $l \geq 22$ will do.

This vector-valued inequality automatically yields the vector-valued inequality of the maximal operator of the singular integral appearing in [3]. We will quote the definition of singular integral from [8].

Definition 1.1 We say that $\mu$ satisfies the growth condition if

$$
\mu(B(x, r)) \leq C r^{n} \text { for all } r>0 .
$$

Here $n$ is a positive constant that can be different from the (geometric or Euclidean) dimension of $X$.

Definition 1.2 Let $\mu$ and $n$ be as above, the singular integral operator is a bounded linear operator $T: L^{2}(X) \rightarrow L^{2}(X)$ that satisfies the following:

There exists a function $K$ that satisfies three properties listed below.

(1) There exists $C>0$ such that $|K(x, y)| \leq C / d(x, y)^{n}$.

(2) There exist $\epsilon>0$ and $C>0$ such that

$$
|K(x, y)-K(z, y)|+|K(y, x)-K(y, z)| \leq C \frac{d(x, z)^{\epsilon}}{d(x, y)^{\epsilon+n}},
$$

if $d(x, y)>2 d(x, z)$.

(3) If $f$ is a bounded measurable function with a bounded support, then we have

$$
T f(x)=\int_{X} K(x, y) f(y) d \mu(y) \quad \text { for all } x \notin \operatorname{supp}(f) .
$$

Definition 1.3 We also define the maximal operator of the truncated integral by the formula 


$$
T^{*} f(x)=\sup _{r>0}\left|\int_{\{y \in X \mid d(x, y)>r\}} K(x, y) f(y) d \mu(y)\right| .
$$

By using the result of [8] and Theorem 1.3, we get the result of [4] again.

Theorem $1.4[4] \quad$ Let $K, T$ and $\mu$ be ones appearing in the above definitions. If $1<p, q<\infty$, then we have

$$
\left\|\left(\sum_{j \in \mathbf{Z}}\left(T^{*} f_{j}\right)^{q}\right)^{1 / q}\right\|_{p} \leq C_{p, q}\left\|\left(\sum_{j \in \mathbf{Z}}\left|f_{j}\right|^{q}\right)^{1 / q}\right\|_{p} .
$$

When we consider the Euclidean space endowed with a standard distance, we have $M_{1}$ is weak- $(1,1)$ bounded. This is due to the Besicovitch covering lemma. For the proof see, for example, [7].

For the proof of that lemma, see [2]. We just cite it below for completeness and comparison with our Theorem 1.5. We state it in the form different from the one stated in [2].

Definition 1.4 Let $\left\{B_{\lambda}\right\}_{\lambda \in L}$ be a family of balls in the metric space. We say $\left\{B_{\lambda}\right\}_{\lambda \in L}$ is disjoint if $B_{\lambda_{1}} \cap B_{\lambda_{2}}=\emptyset$ for $\lambda_{1} \neq \lambda_{2}$. Let $\mathcal{B}$ be a family of balls and $\left\{B_{\lambda}\right\}_{\lambda \in L_{1}}, \ldots,\left\{B_{\lambda}\right\}_{\lambda \in L_{N}}$ be subfamilies of $\mathcal{B}$. We say $\left\{B_{\lambda}\right\}_{\lambda \in L_{1}}, \ldots,\left\{B_{\lambda}\right\}_{\lambda \in L_{N}}$ are disjoint subfamilies if $\left\{B_{\lambda}\right\}_{\lambda \in L_{j}}$ is disjoint for all $j$.

Lemma 1.1 Let $\left\{B_{\lambda}\right\}_{\lambda \in L}$ be a family of balls. Suppose the diameters of balls $\left\{B_{\lambda}\right\}_{\lambda \in L}$ are bounded. Then there exists an integer $N$ depending only on the dimension that has the following property:

There are $N$ disjoint subfamilies $\mathcal{G}_{1}, \mathcal{G}_{2}, \ldots, \mathcal{G}_{N}$ such that all the centers of balls $\left\{B_{\lambda}\right\}_{\lambda \in L}$ belong to a ball in some $\mathcal{G}_{j}$.

Parallel to this we also define the uncentered maximal operator

$$
M_{k, u c} f(x)=\sup _{x \in B(y, r)} \frac{1}{\mu(B(y, k r))} \int_{B(y, r)}|f(z)| d \mu(z) .
$$

We devote Section 3 to the study of $M_{k, u c}$. Similar example appearing in Proposition 1.1 shows that there exist $(X, d)$ and $\mu$ such that $M_{k, u c}$ is bounded if and only if $k \geq 3$. In Euclidean setting, endowed with a standard distance, $M_{1}$ is weak- $(1,1)$ is bounded by Besicovitch covering lemma. But as for the uncentered version, $M_{k, u c}$ is bounded only if $k>1$. We show this by proving a new covering lemma (Theorem 1.5). 
Theorem 1.5 For all $k>1$ there exists an integer $N=N_{k}$, depending only on the dimension and $k$, that satisfies the following:

Let $\left\{B\left(x_{\lambda}, r_{\lambda}\right)\right\}_{\lambda \in L}$ be a family of balls in Euclidean space endowed with a standard distance. Suppose that $\sup _{\lambda \in L} r_{\lambda}<\infty$.

Then we can take disjoint subfamilies

$$
\left\{B\left(x_{\rho}, r_{\rho}\right)\right\}_{\rho \in L_{1}},\left\{B\left(x_{\rho}, r_{\rho}\right)\right\}_{\rho \in L_{2}}, \ldots,\left\{B\left(x_{\rho}, r_{\rho}\right)\right\}_{\rho \in L_{N}}
$$

such that $\bigcup_{\lambda \in L} B\left(x_{\lambda}, r_{\lambda}\right) \subset \bigcup_{j=1, \ldots, N} \bigcup_{\rho \in L_{j}} B\left(x_{\rho}, k r_{\rho}\right)$.

Using this theorem, we can prove $M_{k, u c}$ is weak- $(1,1)$ bounded, if $k>$ 1. This result is shown by Tolsa [10], where balls are replaced by cubes and the result is shown by using Besicovitch's covering lemma.

Theorem 1.6 $M_{k, u c}$ is bounded, if $k>1$.

As an application of Theorem 1.5, we also obtain

Theorem 1.7 If $1<p<\infty, 1<q \leq \infty$ and $1<k<\infty$, then we have

$$
\left\|\left(\sum_{j \in \mathbf{Z}}\left(M_{k, u c} f_{j}\right)^{q}\right)^{1 / q}\right\|_{p} \leq C_{p, q, k}\left\|\left(\sum_{j \in \mathbf{Z}}\left|f_{j}\right|^{q}\right)^{1 / q}\right\|_{p} .
$$

For the uncentered version with $k=1$, see [6]: There exists a measure such that $M_{1, u c}$ is not weak- $(1,1)$ bounded.

\section{The centered maximal operator}

\subsection{A covering lemma}

The first covering lemma is the refinement of the Vitali's covering lemma, which leads us to obtain the weak- $(1,1)$ boundedness of $M_{2}$. And it is used in application again.

Lemma 2.1 Let $\delta>0$. Suppose we have a family of $n$ balls $\left\{B\left(x_{j}, r_{j}\right)\right\}_{j \in\{1, \ldots, n\}}$. Then we can take a subfamily $\left\{B\left(x_{j}, r_{j}\right)\right\}_{j \in A}$ such that

(1) $\left\{B\left(x_{j}, r_{j}\right)\right\}_{j \in A}$ is disjoint.

(2) $\bigcup_{j \in\{1, \ldots, n\}} B\left(x_{j}, \delta r_{j}\right) \subset \bigcup_{j \in A} B\left(x_{j},(2+\delta) r_{j}\right)$.

Remark 2.1 This is an extension of the Vitali's covering lemma: The lemma is precisely Vitali's covering lemma if $\delta=1$. 
Proof. We select $j_{1}$ so that $r_{j_{1}}=\max \left\{r_{1}, \ldots, r_{n}\right\}$. If

$$
\bigcup_{j \in\{1, \ldots, n\}} B\left(x_{j}, \delta r_{j}\right) \subset B\left(x_{j_{1}},(2+\delta) r_{j_{1}}\right),
$$

we have nothing else to do. Let us assume otherwise in the sequel. We define

$$
\Lambda_{1}=\left\{j \in\{1, \ldots, n\} \mid \begin{array}{c}
B\left(x_{j}, \delta r_{j}\right) \text { isn’t contained in } \\
B\left(x_{j_{1}},(2+\delta) r_{j_{1}}\right)
\end{array}\right\} .
$$

We inductively define the subsets of $\{1, \ldots, n\}$ and $j_{1}, \ldots, j_{p} \in\{1, \ldots, n\}$ as follows:

Suppose that $j_{1}, \ldots, j_{q-1}$ and the subsets $\Lambda_{1}, \ldots, \Lambda_{q-1} \subset\{1, \ldots, n\}$ are defined. Then we take $j_{q}$ so that

$$
r_{j_{q}}=\max _{j \in \Lambda_{1} \cap \cdots \cap \Lambda_{q-1}} r_{j} \text { with } j_{q} \in \Lambda_{1} \cap \cdots \cap \Lambda_{q-1}
$$

and we define

$$
\Lambda_{q}=\left\{j \in\{1, \ldots, n\} \mid \begin{array}{c}
B\left(x_{j}, \delta r_{j}\right) \text { isn’t contained in } \\
\bigcup_{j=j_{1}, \ldots, j_{q-1}} B\left(x_{j},(2+\delta) r_{j}\right)
\end{array}\right\} .
$$

This procedure will be stopped because we are dealing with the finite number of the balls. Suppose we have stopped after we selected $j_{p}$ and $\Lambda_{p}$. We will verify that $A=\left\{j_{1}, \ldots, j_{p}\right\}$ satisfies all the requirement of the lemma.

To verify this we fix $j \in\{1, \ldots, n\}$. We have three possibilities.

(a) $j \in\left\{j_{1}, \ldots, j_{p}\right\}$.

(b) $r_{j}=r_{j_{p}}$ and $j \notin\left\{j_{1}, \ldots, j_{p}\right\}$.

(c) $r_{j_{k}} \geq r_{j}>r_{j_{k+1}}$ for some $k \in\{1, \ldots, p-1\}$ and $j \notin\left\{j_{1}, \ldots, j_{p}\right\}$.

We want to show that $B\left(x_{j}, \delta r_{j}\right) \subset \cup_{j \in A} B\left(x_{j},(2+\delta) r_{j}\right)$. If (a) happens, this inclusion is clear. We assume (c) in the sequel. The rest of the possibility can be dealt similarly. Assuming (c) we have $B\left(x_{j}, \delta r_{j}\right) \subset$ $\bigcup_{j \in\left\{j_{1}, j_{2}, \ldots, j_{k}\right\}} B\left(x_{j},(2+\delta) r_{j}\right)$ by the definition of $\Lambda_{1}, \ldots, \Lambda_{k}$ and $r_{j_{k+1}}$. Thus our claim is justified.

Moreover the balls $\left\{B\left(x_{j}, r_{j}\right)\right\}_{j \in A}$ are disjoint. Indeed suppose $k<k^{\prime}$, so that we have $r_{j_{k}} \geq r_{j_{k^{\prime}}}$. By the definition of $\Lambda_{j}$, we have $B\left(x_{j_{k^{\prime}}}, \delta r_{j_{k^{\prime}}}\right)$ is not contained in $B\left(x_{j_{k}},(2+\delta) r_{j_{k}}\right)$, since $j_{k}<j_{k^{\prime}}$. Thus the center $x_{j_{k^{\prime}}}$ is not an element of $B\left(x_{j_{k}}, 2 r_{j_{k}}\right)$. This implies $d\left(x_{j_{k}}, x_{j_{k^{\prime}}}\right) \geq 2 r_{j_{k}}$. Furthermore 
as noted, we have $r_{j_{k}} \geq r_{j_{k}^{\prime}}$. Combining them, we obtain $\left\{B\left(x_{j}, r_{j}\right)\right\}_{j \in A}$ is disjoint.

\subsection{Proof of Theorem 1.2}

First of all let us remark the following fact, which is often used in the sequel.

Remark 2.2 $B(x, r)$ is an open ball with radius $r>0$ and center $x \in X$. We use $\bar{B}(x, r)$ to denote a closed ball with radius $r>0$ and center $x \in X$. By Radon property, we can replace $B(x, r)$ by $\bar{B}(x, r)$ in the definition of $M_{k}$ and $M_{k, u c}$. Thus for all measurable $f: X \rightarrow \mathbf{C}$ we have $M_{k} f(x) \rightarrow$ $M_{k_{0}} f(x)$ as $k \rightarrow k_{0}$.

Noting this remark, we shall prove Theorem 1.2. Fix $\lambda>0$. By Remark 2.2 , it follows that

$$
\bigcup_{k>2}\left\{x \in X \mid M_{k} f(x)>\lambda\right\}=\left\{x \in X \mid M_{2} f(x)>\lambda\right\} .
$$

Let $\delta>0$ and $k=2+\delta$. We define $E_{k}=\left\{x \in X \mid M_{k} f(x)>\lambda\right\}$. For all $x \in E_{k}$, by its definition there exists $r_{x}>0$ such that

$$
\frac{1}{\mu\left(B\left(x, k r_{x}\right)\right)} \int_{B\left(x, r_{x}\right)}|f(y)| d \mu(y)>\lambda .
$$

Since $\mu$ is a Radon measure, $E_{k}$ is an open set. Since $X$ is separable, so with the aid of the Linderöf covering theorem we can take $x_{j} \in E_{k}, j=1,2, \ldots$ such that $E_{k} \subset \bigcup_{j \in \mathbf{N}} B\left(x_{j}, \delta r_{x_{j}}\right)$.

By Lemma 2.1 there exists $A \subset\{1, \ldots, n\}$ such that

$$
\begin{array}{r}
\bigcup_{j=1, \ldots, n} B\left(x_{j}, \delta r_{x_{j}}\right) \subset \bigcup_{l \in A} B\left(x_{l},(2+\delta) r_{x_{l}}\right) \text { and } \\
\left\{B\left(x_{l}, r_{x_{l}}\right)\right\}_{l \in A} \text { is disjoint. }
\end{array}
$$

By the definition of $E_{k}$, we also have

$$
\mu\left(B\left(x_{l},(2+\delta) r_{x_{l}}\right)\right) \leq \frac{1}{\lambda} \int_{B\left(x_{l}, r_{x_{l}}\right)}|f(x)| d \mu(x) .
$$

Putting them together, we obtain

$$
\mu\left(\bigcup_{j=1,2, \ldots, n} B\left(x_{j}, \delta r_{x_{j}}\right)\right)
$$




$$
\begin{aligned}
& \leq \mu\left(\bigcup_{l \in A} B\left(x_{l},(2+\delta) r_{x_{l}}\right)\right) \leq \sum_{l \in A} \mu\left(B\left(x_{l},(2+\delta) r_{x_{l}}\right)\right) \\
& \leq \sum_{l \in A} \frac{1}{\lambda} \int_{B\left(x_{l}, r_{x_{l}}\right)}|f(x)| d \mu(x) \leq \frac{1}{\lambda} \int_{X}|f(x)| d \mu(x) .
\end{aligned}
$$

Letting $n$ tend to infinity in the above inequality,

$$
\mu\left(E_{k}\right) \leq \frac{1}{\lambda} \int_{X}|f(x)| d \mu(x)
$$

is derived. As is noted in Remark 2.2, we have

$$
\bigcup_{k>2}\left\{x \in X \mid M_{k} f(x)>\lambda\right\}=\left\{x \in X \mid M_{2} f(x)>\lambda\right\} .
$$

Tending $k \downarrow 2$, we get

$$
\mu\left(\left\{x \in X \mid M_{2} f(x)>\lambda\right\}\right) \leq \frac{1}{\lambda} \int_{X}|f(x)| d \mu(x) .
$$

Remark 2.3 This theorem may be regarded as some extension of the result [1]. In [1], H. Carlsson proved his result on the Euclidean space with Lebesgue measure with Euclidian distance. He used the method appeared in [5]. But we cannot apply it to the metric space with non-doubling measure, because we cannot use the Lebesgue differentiation theorem in general separable metric spaces. Another proof using an outer measure and related results can be found in [9].

Corollary 2.1 We have for $p>1\left\|M_{2} f\right\|_{p} \leq C_{p}\|f\|_{p}$.

Proof. Since $\left\|M_{2} f\right\|_{\infty} \leq\|f\|_{\infty}$ is trivial, by interpolation we obtain the desired inequality.

\subsection{An example showing sharpness of Theorem 1.2}

Next we want to construct a space where $M_{k}$ is not bounded if $k<2$. First we define a set on which the distance and the measure will be defined. The distance is quite different from the one of the usual Euclidean space. We will define $D, X$ and so on. They are valid only in this subsection.

Definition 2.1 Let $D$ be a closed unit disk on the complex plane. Define $X$ as a direct product of a countable copies of $D$.

In what follows $[\cdot]$ is used to denote the Gauss sign. 
Definition 2.2 We define a function $d$ as follows: Take $\mathbf{x}=\left\{x_{n}\right\}_{n \in \mathbf{N}}$ and $\mathbf{y}=\left\{y_{n}\right\}_{n \in \mathbf{N}}$. Then there exists $r \leq 2$ such that $\left|x_{n}-y_{n}\right| \leq r$ for all $n \leq 2+\left[\log _{10}(1 / r)\right]$ We define $d(\mathbf{x}, \mathbf{y})$ as an infimum of such $r>0$.

Remark 2.4 $r$ that appears in the definition does exist: $r=2$ will be enough.

Lemma 2.2 This function defines a distance.

Proof. We will omit the proof, since it is easy to show the lemma, noting next remark.

Remark 2.5 If $r>0$, the open ball $B(x, r)$ is precisely the set

$$
\left\{\mathbf{y}=\left\{y_{n}\right\}_{n \in \mathbf{N}} \in X|| x_{n}-y_{n} \mid<r \text { for all } n \leq 2+\left[\log _{10} \frac{1}{r}\right]\right\} \text {. }
$$

Lemma 2.3 The space $X$, endowed with a distance function $d$, is a separable subspace.

Proof. Using standard argument, this lemma is easy to show. So we will omit the proof.

This is the distance space we work on. Next let us define the measure.

Definition 2.3 Let $\mathcal{B}$ be a $\sigma$-algebra generated by $B(\mathbf{x}, r)$ with $\mathbf{x} \in X$ and $r>0$.

The following proposition ensures the measurability of a maximal function.

Proposition 2.1 The $\sigma$-algebra $\mathcal{B}$ is nothing but the one generated by the cylinder sets of the form $A_{1} \times \cdots \times A_{l} \times D \times D \times \cdots$, where $A_{1}, \ldots, A_{l}$ are Bore-measurable sets of $D$.

Proof. Put $\Delta(a, r)=\{z \in D|| z-a \mid<r\}$, where $a \in D$ and $r>0$. Let us show that $\Delta\left(a_{1}, r_{1}\right) \times \Delta\left(a_{2}, r_{2}\right) \times \cdots \times \Delta\left(a_{l}, r_{l}\right) \times D \times D \times \cdots$ is $\mathcal{B}$-measurable, if $a_{1}, a_{2}, \ldots, a_{l} \in D$ and $r_{1}, r_{2}, \ldots, r_{l}>0$. In fact $B\left(\mathbf{a}, 10^{-l+2}\right)$ is contained in $\mathcal{B}$ for all $\mathbf{a} \in X$. Since $\Delta\left(a_{1}, r_{1}\right) \times \Delta\left(a_{2}, r_{2}\right) \times \cdots \times \Delta\left(a_{l}, r_{l}\right) \times D \times$ $D \times \cdots$ is expressible as a countable union and intersection of balls of the form $B\left(\mathbf{a}, 10^{-l+2}\right)$, where $\mathbf{a} \in X, \Delta\left(a_{1}, r_{1}\right) \times \Delta\left(a_{2}, r_{2}\right) \times \cdots \times \Delta\left(a_{l}, r_{l}\right) \times D \times$ $D \times \cdots$ is $\mathcal{B}$-measurable. Thus $\sigma$-algebra $\mathcal{B}$ contains $\sigma$-algebra generated by cylinder sets. The reverse inclusion is clear so our claim is justified. 
Remark 2.6 The topology induced by this distance is the same as product topology induced by Euclidean topology of $D$. This can be shown using the same idea as that of Proposition 2.1.

Definition 2.4 We define $f: D \rightarrow \mathbf{R}$ as follows:

Firstly we define $a_{n}=\prod_{j=1}^{n+2} j$ !. We define annulus $A_{n}$ and $B_{n}$ with $n=$ $0,1, \ldots$ as

$$
\begin{aligned}
& A_{n}=\left\{z \in D\left|3^{-n}\left(1-\frac{1}{a_{n}^{3}}\right)<\right| z \mid<3^{-n}\right\} \\
& B_{n}=\left\{z \in D\left|3^{-n-1}<\right| z \mid<3^{-n}\left(1-\frac{1}{a_{n}^{3}}\right)\right\} .
\end{aligned}
$$

And we define the density function $f$ as

$$
f(z)= \begin{cases}l / a_{n} & \text { on } A_{n} \\ l / a_{n+3} 5 & \text { on } B_{n} \\ 0 & \text { otherwise. }\end{cases}
$$

We define $\nu=f(z) d z$, where $d z$ is the Lebesgue measure on $D$. Constant $l$ is taken so that $\nu(D)=1$. Let $\mu_{n}$ be a measure on $D \times D \times \cdots \times D$ (n-tuple) defined as $\mu_{n}=\nu \times \nu \times \cdots \times \nu$. Since $\nu(D)=1$, we can use Kolmogorov's extension theorem to define $\mu$ as a countable product of $\nu$ : $\mu=\lim _{n \rightarrow \infty} \mu_{n}$.

Firstly, let us examine the property of the (complicated) function $f$. It is summarized as a lemma below.

Lemma 2.4 (a) The ball is non-degenerate if its radius is positive. And there are infinitely many integers $n$ such that $B\left(\mathbf{x}, 1 / 3^{n}\right)$ and $B(\mathbf{x}, 1 /(2$. $\left.3^{n}\right)$ ) are made up of the product of the same number of nontrivial balls and the closed unit disks, where a ball is nontrivial means that it is a proper subset of $D$.

(b) Define $\Omega_{1}=\Omega_{1, n}$ as

$$
\Omega_{1}=\left\{x+\sqrt{-1} y \in D \mid\left(x-3^{-n}\right)^{2}+y^{2} \leq 4 \cdot 9^{-n}\right\} .
$$

Then we have

$$
\lim _{n \rightarrow \infty} \frac{\nu\left(\Omega_{1}\right)}{2 \pi l / 9^{n} a_{n}{ }^{4}}=1 .
$$


(c) Let $3 / 2<k<2$. Define $\Omega_{2}=\Omega_{2, n}$ as

$$
\Omega_{2}=\left\{x+\sqrt{-1} y \in D \mid\left(x-3^{-n}\right)^{2}+y^{2} \leq k^{2} \cdot 9^{-n}\right\} .
$$

Then we have

$$
\lim _{n \rightarrow \infty} \frac{\nu\left(\Omega_{2}\right)}{2 \pi l / 9^{n} a_{n}{ }^{4}}=C_{k},
$$

where $C_{k}$ is a geometric constant strictly less than 1 that depends on $k$.

(d) Define $\Omega_{3}=\Omega_{3, n}$ as

$$
\Omega_{3}=\left\{x+\sqrt{-1} y \in D \mid x^{2}+y^{2} \leq 9^{-n}\right\} .
$$

Then we have

$$
\lim _{n \rightarrow \infty} \frac{\nu\left(\Omega_{3}\right)}{2 \pi l / 9^{n} a_{n}{ }^{4}}=1 .
$$

Proof. In view of Remark 2.5, (a) is clear because $\log _{10} 3=0.4771 \cdots$ and $f(x)$ is $d x$-almost everywhere positive, where $d x$ is a Lebesgue measure on $D$. Let us prove (b). Note that $\left\{x+\sqrt{(-1)} y \in D \mid x^{2}+y^{2} \leq 9^{-n}\right\} \subset \Omega_{1} \subset$ $\left\{x+\sqrt{(-1)} y \in D \mid x^{2}+y^{2} \leq 9^{1-n}\right\}$.

Hence we have $\Omega_{1} \cap A_{k}=A_{k}, \Omega_{1} \cap B_{k}=B_{k}$, if $n \leq k$ and that $\Omega_{1} \cap$ $A_{k}=\emptyset, \Omega_{1} \cap B_{k}=\emptyset$, if $n>k+1$. Taking this into account, we will estimate $\Omega_{1} \cap A_{k}$ with $n<k, \Omega_{1} \cap B_{k}$ with $n<k, \Omega_{1} \cap A_{n}, \Omega_{1} \cap B_{n}$, $\Omega_{1} \cap A_{n-1}$, and $\Omega_{1} \cap B_{n-1}$ respectively. Firstly a little long computation leads us to a rough estimate that $\nu\left(\Omega_{1} \cap A_{n-1}\right)$ is bounded by $C / 9^{n} a_{n-1}{ }^{6}$, which is less than $C / 9^{n} a_{n}{ }^{4}$ if $n$ is large. As for $\nu\left(\Omega_{1} \cap B_{n-1}\right)$, it is bounded by $C / 9^{n} a_{n+2}{ }^{5}$, which is also less than $C / 9^{n} a_{n}{ }^{4}$ if $n$ is large. Furthermore we have $\lim _{n \rightarrow \infty}\left(\nu\left(\Omega_{1} \cap A_{n}\right)\right) /\left(2 \pi l / 9^{n} a_{n}{ }^{4}\right)=1$. This is due to the fact that the Lebesgue measure of $A_{n}$ is equal to

$$
\pi\left(3^{-n}\right)^{2}-\pi\left(3^{-n}\left(1-\frac{1}{a_{n}{ }^{3}}\right)\right)^{2} \approx \frac{2 \pi}{9^{n} a_{n}{ }^{3}}
$$

and the fact that we have $f(x)=l / a_{n}$.

We also have

$$
\nu\left(\Omega_{1} \cap \bigcup_{k: n<k} A_{k}\right) \leq \frac{C}{9^{n} a_{n+1}{ }^{3} a_{n}}
$$

and 


$$
\nu\left(\Omega_{1} \cap \bigcup_{k: n \leq k} B_{k}\right) \leq \frac{C}{9^{n} a_{n+3}{ }^{5} a_{n}} .
$$

With these estimates we obtain (b).

(c) and (d) follow similarly. But the proof of (c) is the crucial point of the proof of unboundedness of $M_{k}$ with $k<2$. So we will point out what counts.

The essential difference lies in the estimate of $\nu\left(\Omega_{2} \cap A_{n}\right)$. With $k$ fixed geometric observation shows that there exists $C_{k}$ strictly less than 1 such that $\nu\left(\Omega_{2} \cap A_{n}\right) / \nu\left(A_{n}\right) \rightarrow C_{k}$ as $n \rightarrow \infty$. More precisely, $C_{k}$ is given by the following formula:

$$
\begin{aligned}
C_{k} & =\frac{\left|\left\{(x, y) \mid x^{2}+y^{2}=1,(x-1)^{2}+y^{2} \leq k^{2}\right\}\right|}{\left|\left\{(x, y) \mid x^{2}+y^{2}=1\right\}\right|} \\
& =\frac{1}{\pi} \cos ^{-1}\left(\frac{2-k^{2}}{2}\right),
\end{aligned}
$$

where $|E|$ means arc length of an arc $E$. This is the critical point of (c) and the rest is quite similar to that of (b), so the detail is omitted.

Remark 2.7 Our calculation shows

$$
\nu\left(\Omega_{1}\right)=\frac{2 \pi l}{9^{n} a_{n}{ }^{4}}\left(1+O\left(\frac{1}{n^{2}}\right)\right) .
$$

This will be used in Remark 2.9.

Under this measure, we will show that $M_{k}$ is bounded only if $k \geq 2$. Before proving this, we introduce one more notation.

Notation 2.1 For a positive measure $\alpha$ on $X$ we denote

$$
\sup _{r>0} \frac{\alpha(B(\mathbf{x}, r))}{\mu(B(\mathbf{x}, k r))} \quad \text { by } \quad M_{k} \alpha(\mathbf{x}) \text {. }
$$

Let $\delta_{\mathbf{0}}$ the Dirac measure at $\mathbf{0}=(0,0, \ldots)$.

Proposition 2.2 $M_{k}$ is not bounded, if $k<2$.

Proof. We may assume $3 / 2<k<2$, since $M_{k}$ is decreasing as $k$ increases. Suppose we have $M_{k}$ is bounded. We want to derive a contradiction. We begin with constructing an approximation of Dirac delta. Let $g_{r}=1_{B(\mathbf{0}, r)} / \mu(B(\mathbf{0}, r)) . M_{k} g_{r}$ tends pointwise to $M_{k} \delta_{\mathbf{0}}$ as $r \rightarrow 0$, where $\delta_{\mathbf{0}}$ is a point mass at $\mathbf{0}$. Let $\mathbf{a}$ be a point defined as follows: 
Put $K_{n}=2+\left[\log _{10} 3^{n}\right]$. Define $\mathbf{a}=\mathbf{a}_{\mathbf{n}}=\left(a_{j}\right)_{j \in \mathbf{N}} \in D$ as

$$
a_{j}= \begin{cases}3^{-n} & \left(j \leq K_{n}\right) \\ 0 & \text { (otherwise) }\end{cases}
$$

Take $\lambda=1 / \mu\left(B\left(\mathbf{a}, k 3^{-n}\right)\right)$. We have $\left\{x \in X \mid M_{k} \delta_{\mathbf{0}}(x)>\lambda\right\} \supset$ $B\left(\mathbf{0}, 3^{-n}\right)$. Thus we have $\mu\left(B\left(\mathbf{0}, 3^{-n}\right)\right) \leq C / \lambda$, that is,

$\mu\left(B\left(\mathbf{0}, 3^{-n}\right)\right) / \mu\left(B\left(\mathbf{a}, k 3^{-n}\right)\right) \leq C$. By the definition of $\nu$ and Lemma $2.4(\mathrm{~b})$, there are infinitely many integers $n$ such that

$$
\left\{\frac{\nu\left(\left\{x+\sqrt{-1} y \in D \mid x^{2}+y^{2} \leq 9^{-n}\right\}\right)}{\nu\left(\left\{x+\sqrt{-1} y \in D \mid\left(x-3^{-n}\right)^{2}+y^{2} \leq k^{2} \cdot 9^{-n}\right\}\right)}\right\}^{\left(2+\left[\log _{10} 3^{n}\right]\right)} \leq C .
$$

Take limit of this quantity as n, running through such a integer, to infinity. With the aid of (c) and (d) of Lemma 2.4 contradiction is obtained, since $C_{k}$ is strictly less than 1 .

By construction we can check the following:

Proposition 2.3 In the above space the measure of $B(x, r)$ grows in the polynomial order of any degree, that is, $\mu(B(x, r)) / r^{n}$ is bounded for all positive integer $n$.

Remark 2.8 This proposition can be interpreted that the dimension of the space is "infinity" according to the terminology of [8]. But this proposition cannot be improved in the sense that $\mu(B(x, r)) / r^{n}$ is bounded uniformly on $n$.

Remark 2.9 Using Lemma 2.4(b), (d) and Remark 2.7, the proof of the Theorem 1.2 and the reproduction of the proof with the parameter of $k$ changed into 2 shows that the Theorem 1.2 is sharp in the following sense: We cannot take the weak- $(1,1)$ constant strictly less than 1 in general.

\subsection{Proof of Theorem 1.3 and Theorem 1.4}

As an application of Lemma 2.1 and Theorem 1.2 we will prove Theorem 1.3 and Theorem 1.4. First of all we get a weighted inequality of the Stein type, using again Lemma 2.1.

Proposition 2.4 We have

$$
\int_{\left\{M_{7} f>\lambda\right\}}|g(x)| d \mu(x) \leq \frac{1}{\lambda} \int_{X}|f(x)| M_{2} g(x) d \mu(x) .
$$


Proof. Take $k>7$. Let $E=\left\{x \in X \mid M_{k} f>\lambda\right\}$. By the definition of $E$, for all $x \in E$ there exists $r_{x}$ such that

$$
\frac{1}{\mu\left(B\left(x, k r_{x}\right)\right)} \int_{B\left(x, r_{x}\right)}|f(x)| d \mu(x)>\lambda .
$$

Since $E$ is an open set, again by the Linderöf theorem, there exist $x_{j}, j=1,2, \ldots$ such that $E \subset \cup_{j \in \mathbf{N}} B\left(x_{j}, a r_{x_{j}}\right)$. We will take $a=(k-$ 7) $/ 20$.

We claim that

$$
\int_{\bigcup_{j=1, \ldots, n} B\left(x_{j}, a r_{x_{j}}\right)}|g(x)| d \mu(x) \leq \frac{1}{\lambda} \int_{X}|f(x)| M_{2} g(x) d \mu(x) .
$$

By Lemma 2.1 there exists a subfamily of balls $\left\{B\left(x_{j}, a r_{x_{j}}\right)\right\}_{j \in \Lambda}$ with $(\Lambda \subset\{1,2, \ldots, n\})$ that satisfies the following properties.

(a) $\left\{B\left(x_{j}, r_{x_{j}}\right)\right\}_{j \in \Lambda}$ is disjoint.

(b) $\bigcup_{j=1, \ldots, n} B\left(x_{j}, a r_{x_{j}}\right) \subset \bigcup_{j \in \Lambda} B\left(x_{j},(2+a) r_{x_{j}}\right)$.

Note that we have, for all $x \in B\left(x_{j}, r_{x_{j}}\right)$,

$$
\begin{aligned}
& \frac{1}{\mu\left(B\left(x_{j}, k r_{x_{j}}\right)\right)} \int_{B\left(x_{j},(2+a) r_{x_{j}}\right)}|g(y)| d \mu(y) \\
\leq & \frac{1}{\mu\left(B\left(x,(6+2 a) r_{x_{j}}\right)\right)} \int_{B\left(x,(3+a) r_{x_{j}}\right)}|g(y)| d \mu(y) \leq M_{2} g(x) .
\end{aligned}
$$

Using this, we obtain

$$
\begin{aligned}
& \int_{\bigcup_{j=1, \ldots, n} B\left(x_{j}, a r_{x_{j}}\right)}|g(x)| d \mu(x) \\
\leq & \int_{\bigcup_{j \in \Lambda} B\left(x_{j},(2+a) r_{x_{j}}\right)}|g(x)| d \mu(x) \leq \sum_{j \in \Lambda} \int_{B\left(x_{j},(2+a) r_{x_{j}}\right)}|g(x)| d \mu(x) \\
\leq & \sum_{j \in \Lambda} \frac{1}{\lambda} \int_{B\left(x_{j}, r_{x_{j}}\right)}|f(x)| d \mu(x) \\
& \times \frac{1}{\mu\left(B\left(x_{j}, k r_{x_{j}}\right)\right)} \int_{B\left(x_{j},(2+a) r_{x_{j}}\right)}|g(x)| d \mu(x) \\
\leq & \sum_{j \in \Lambda} \frac{1}{\lambda} \int_{B\left(x_{j}, r_{x_{j}}\right)}|f(x)| M_{2} g(x) d \mu(x) \leq \frac{1}{\lambda} \int_{X}|f(x)| M_{2} g(x) d \mu(x) .
\end{aligned}
$$


By the definition of $E$ we have

$$
\int_{\left\{M_{k} f>\lambda\right\}}|g(x)| d \mu(x) \leq \frac{1}{\lambda} \int_{X}|f(x)| M_{2} g(x) d \mu(x) .
$$

Letting $k \downarrow 7$, we finally obtain

$$
\int_{\left\{M_{7} f>\lambda\right\}}|g(x)| d \mu(x) \leq \frac{1}{\lambda} \int_{X}|f(x)| M_{2} g(x) d \mu(x) .
$$

Thus we have finished.

Corollary 2.2 If $p>1$, then we have

$$
\int_{X}\left(M_{7} f(x)\right)^{p}|g(x)| d \mu(x) \leq C_{p} \int_{X}|f(x)|^{p} M_{2} g(x) d \mu(x) .
$$

Proof. For a positive function $w$ we denote $\|\cdot\|_{\infty, w}$ is a $L^{\infty}$-norm of the function with respect to the weighted measure $w d \mu$. Since

$$
\left\|M_{7} f\right\|_{\infty,|g|} \leq\|f\|_{\infty, M_{2} g}
$$

is clear, this is again just a matter of the interpolation of this inequality and the last results.

Remark 2.10 We use the following analogous which is used below to obtain Theorem 1.3. The proof is only the change of the parameters $k$ of Proposition 2.4 and Corollary 2.2 respectively.

Proposition 2.5 Let $X, \mu$ be as above.

(a) The estimate

$$
\int_{\left\{M_{22} f>\lambda\right\}}|g(x)| d \mu(x) \leq \frac{1}{\lambda} \int_{X}|f(x)| M_{7} g(x) d \mu(x)
$$

holds for all $\lambda>0$.

(b) If $p>1$, then we have

$$
\int_{X}\left(M_{22} f(x)\right)^{p}|g(x)| d \mu(x) \leq C_{p} \int_{X}|f(x)|^{p} M_{7} g(x) d \mu(x) .
$$

At last we are in the position of proving Theorem 1.3. If $p \geq q>1$, a little more can be said. We have the following. 
Theorem 2.1 If $p \geq q>1$, then

$$
\left\|\left(\sum_{j \in \mathbf{Z}}\left(M_{7} f_{j}\right)^{q}\right)^{1 / q}\right\|_{p} \leq C_{p, q}\left\|\left(\sum_{j \in \mathbf{Z}}\left|f_{j}\right|^{q}\right)^{1 / q}\right\|_{p} .
$$

Proof. The case when $p=q$ is trivial. Assume that $q<p$. Put $r=p / q$ and let $r^{\prime}$ be a conjugate exponent of $r$.

By using Theorem 1.2 and Corollary 2.2 we get to

$$
\begin{aligned}
& \left\|\left(\sum_{j \in \mathbf{Z}}\left(M_{7} f_{j}\right)^{q}\right)^{1 / q}\right\|_{p}^{q}=\sup _{\|g\|_{r^{\prime}}=1, g \geq 0} \int\left(\sum_{j \in \mathbf{Z}}\left(M_{7} f_{j}\right)^{q}\right) g d \mu \\
= & \sup _{\|g\|_{r^{\prime}}=1, g \geq 0} \sum_{j \in \mathbf{Z}} \int\left(M_{7} f_{j}\right)^{q} g d \mu \leq C_{p, q} \sup _{\|g\|_{r^{\prime}}=1, g \geq 0} \sum_{j \in \mathbf{Z}} \int\left|f_{j}\right|^{q} M_{2} g d \mu \\
\leq & C_{p, q} \sup _{\|g\|_{r}^{\prime}=1, g \geq 0}\left\{\int\left(\sum_{j \in \mathbf{Z}}\left|f_{j}\right|^{q}\right)^{r} d \mu\right\}^{1 / r}\left\{\int\left(M_{2} g\right)^{r^{\prime}} d \mu\right\}^{1 / r^{\prime}} \\
\leq & C_{p, q}\left\{\int\left(\sum_{j \in \mathbf{Z}}\left|f_{j}\right|^{q}\right)^{r} d \mu\right\}^{1 / r} \sup _{\|g\|_{r}^{\prime}=1, g \geq 0}\left(\int g^{r^{\prime}} d \mu\right)^{1 / r^{\prime}} \\
= & C_{p, q}\left\{\int\left(\sum_{j \in \mathbf{Z}}\left|f_{j}\right|^{q}\right)^{r} d \mu\right\}^{1 / r} .
\end{aligned}
$$

Taking $1 / q$-th power of both sides, we obtain

$$
\left\|\left(\sum_{j \in \mathbf{Z}}\left(M_{7} f_{j}\right)^{q}\right)^{1 / q}\right\|_{p} \leq C_{p, q}\left\|\left(\sum_{j \in \mathbf{Z}}\left|f_{j}\right|^{q}\right)^{1 / q}\right\|_{p} .
$$

For the case that $q>p$, we use Proposition 2.5 and Theorem 2.1

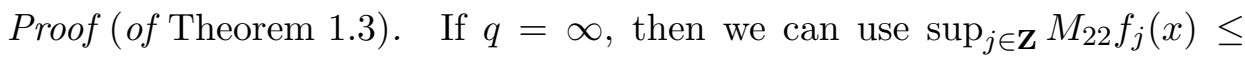
$M_{22}\left(\sup _{j \in \mathbf{Z}}\left|f_{j}\right|\right)(x)$. Hence the theorem is clear, if $q=\infty$. By Theorem 2.1 , it remains to show when $p<q<\infty$. Let $q>p$ in what follows and take another $r<q$ so close to $q$ that $p r / q>1$. According to $\left(L^{p r / q}-L^{(p r / q)^{\prime}}\right)$ duality we have

$$
\left\|\left(\sum_{j \in \mathbf{Z}}\left(M_{22} f_{j}\right)^{q}\right)^{1 / q}\right\|_{p}^{q / r}=\sup _{\left\{\|g\|_{(p r / q)^{\prime}}=1, g \geq 0\right\}} \int\left(\sum_{j \in \mathbf{Z}}\left(M_{22} f_{j}\right)^{q}\right)^{1 / r} g d \mu
$$

Keeping this in mind, let us fix positive $g$ with $\|g\|_{(p r / q)^{\prime}}=1$. 
Note that $q>p$ implies $(p r / q)^{\prime}>r^{\prime}$, so that we are in the position of using Theorem 2.1 with parameter $(p r / q)^{\prime}>r^{\prime}$. We also use Proposition 2.5 to obtain

$$
\begin{aligned}
& \int\left(\sum_{j \in \mathbf{Z}}\left(M_{22} f_{j}\right)^{q}\right)^{1 / r} g d \mu \\
= & \sup _{\sum_{k} h_{k}{ }^{\prime}=1} \int\left(\sum_{k} \geq 0\right. \\
& \left.\left(\left(M_{22} f_{j}\right)^{q / r} h_{j}\right) g\right) d \mu
\end{aligned}
$$

(Below we will write sup instead of $\sup _{\sum_{k} h_{k}{ }^{r^{\prime}}=1 h_{k} \geq 0}$.)

$$
\begin{aligned}
\leq & C_{p, q} \sup \int\left(\sum_{j \in \mathbf{Z}}\left|f_{j}\right|^{q / r} M_{7}\left(h_{j} g\right)\right)^{\prime} d \mu \\
\leq & C_{p, q} \sup \int\left(\sum_{j \in \mathbf{Z}}\left|f_{j}\right|^{q}\right)^{1 / r}\left(\sum_{j \in \mathbf{Z}}\left(M_{7} h_{j} g\right)^{r^{\prime}}\right)^{1 / r^{\prime}} d \mu \\
\leq & C_{p, q} \sup \left\{\int\left(\sum_{j \in \mathbf{Z}}\left|f_{j}\right|^{q}\right)^{p / q} d \mu\right\}^{q / p r} \\
& \times\left\{\int\left(\sum_{j \in \mathbf{Z}}\left(M_{7} h_{j} g\right)^{r^{\prime}}\right)^{(p r / q)^{\prime} / r^{\prime}} d \mu\right\}^{1 /(p r / q)^{\prime}} \\
\leq & C_{p, q}\left\{\int\left(\sum_{j \in \mathbf{Z}}\left|f_{j}\right|^{q}\right)^{p / q} d \mu\right\}^{q / p r} \\
& \times \sup \left\{\int\left(\sum_{j \in \mathbf{Z}}\left(\left|h_{j} g\right|^{r^{\prime}}\right)\right)^{(p r / q)^{\prime} / r^{\prime}} d \mu\right\}^{1 /(p r / q)^{\prime}} \\
= & C_{p, q}\left\{\int\left(\sum_{j \in \mathbf{Z}}\left|f_{j}\right|^{q}\right)^{p / q} d \mu\right\}^{q / p r} \cdot
\end{aligned}
$$

Putting together this and first observation we finish the proof.

This vector-valued inequality is different from the one that appeared in the [8] only in that we enlarged $k$ by 22 or 7 times not by three times.

We will assume the assumption posed on Definition 1.1 and Definition 1.2 until the end of this section. With minor modification of the results of [8] we obtain

Theorem $2.2[8] \quad$ We have for $\beta>1$ and large $l$ 


$$
T^{*} f(x) \leq C_{\beta, l}\left(M_{l}(T f)(x)\right)+C_{\beta, l}\left\{\left(M_{l}|f|^{\beta}\right)(x)^{1 / \beta}\right\} .
$$

The next result is due to García-Cuerva [3].

Theorem 2.3 [3] If $1<p, q<\infty$, we have

$$
\left\|\left(\sum_{j \in \mathbf{Z}}\left|T f_{j}\right|^{q}\right)^{1 / q}\right\|_{p} \leq C_{p, q}\left\|\left(\sum_{j \in \mathbf{Z}}\left|f_{j}\right|^{q}\right)^{1 / q}\right\|_{p} .
$$

Combining these results and Theorem 1.3, we obtain Theorem 1.4.

\section{The uncentered maximal operator on the Euclidean space}

In this section we examine the uncentered maximal operator. The result by [8] which appeared in Introduction is sharp: We can construct a similar example of the space on which $M_{k, u c}$ is not bounded if $k<3$, using an idea of Section 2.3. Hence in this section we limit ourselves to the space $\mathbf{R}^{d}$ with Euclidean distance. If the space is Euclidean and the ball is defined by a standard distance, we shall show that $M_{k, u c}$ is bounded if $k>1$. This is best possible as [6] shows: As in [6] for $\mu=\exp \left(x^{2}+y^{2}\right) d x d y$ in $\mathbf{R}^{2}, M_{1, u c}$ is not weak- $(1,1)$ bounded.

\subsection{Another covering lemma (Proof of Theorem 1.5)}

We want a substitute of Besicovitch's covering lemma. If $k>3$, we put $N_{k}=1$ and we can use the Vitali covering lemma to prove Theorem 1.5. Hence for the proof of Theorem 1.5, we may assume that $k \leq 3$. This Theorem 1.5 is a covering lemma for our purpose. This may be viewed also as a substitute of Vitali's covering lemma. To prove Theorem 1.5, firstly we prove it by posing another assumption.

Lemma 3.1 Let $\left\{B\left(x_{\lambda}, r_{\lambda}\right)\right\}_{\lambda \in L}$ be a family of balls and assume that

$$
\sup _{\lambda \in L} r_{\lambda}<\sqrt{k} \inf _{\lambda \in L} r_{\lambda}<\infty .
$$

Then we can take disjoint subfamilies as in Theorem 1.5.

Proof. First of all by scaling, we may normalize to have $\sup _{\lambda \in L} r_{\lambda}=1$. (We are working on the Euclidean space. So we are able to multiply the scalar.)

In this part we divide the family of balls. More precisely we proceed as follows: Let $\mathcal{Q}_{0}$ be a family of dyadic cubes of side length 1 . Here we 
are now considering cubes of the form $\mathrm{Q}=\Pi_{j=1}^{d}\left[m_{j}, m_{j}+1\right)$, where $m_{j}$ are integers $(j=1, \ldots, d)$. We abbreviate the dyadic cubes in $\mathcal{Q}_{0}$ to "cubes" for short. Let $\mathrm{Q}^{0}$ be $[0,1)^{d}$. We divide the cubes into subfamily:

If $\vec{m}=\left(m_{1}, m_{2}, \ldots, m_{d}\right)$ is an element of $\{0,1,2,3\}^{d}$, we put

$$
\mathcal{Q}_{\vec{m}}=\left\{\mathrm{Q} \in \mathcal{Q}_{0} \mid \mathrm{Q}-(\vec{p}+\vec{m})=\mathrm{Q}^{0} \text { for some } \vec{p} \in(4 \mathbf{Z})^{d}\right\} .
$$

Next we define $L_{\vec{m}}$ as

$$
L_{\vec{m}}=\left\{\lambda \in L \mid x_{\lambda} \text { is contained in some cube in } \mathcal{Q}_{\vec{m}}\right\} .
$$

Note that the cubes in $\mathcal{Q}_{\vec{m}}$ satisfy the following property: Suppose that $Q$ and $Q^{\prime}$ are both in $\mathcal{Q}_{\vec{m}}$ and that $Q$ and $Q^{\prime}$ are different, then the distance between the two cubes is larger than 3. Hence if the center of $B$ is in $Q$ and the center of $B^{\prime}$ is in $Q^{\prime}$, then $B$ and $B^{\prime}$ are disjoint.

Taking into account of the preceding paragraphs we may assume that all the centers of the balls are in $\mathrm{Q}^{0}$. In fact once this is proved, by the last paragraph we can take the balls satisfying the property of this lemma from $\mathcal{Q}_{\vec{m}}$ for any $\vec{m} \in\{0,1,2,3\}^{d}$. For any $\vec{m} \in\{0,1,2,3\}^{d}$, we obtain families $\mathcal{B}_{\vec{m}}^{(1)}, \ldots, \mathcal{B}_{\vec{m}}^{\left(N_{k}\right)}$. Translation shows the number $N_{k}$ is not dependent on $\vec{m}$. So our desired family is

$$
\bigcup_{\vec{m} \in\{0,1,2,3\}^{d}, j \leq N_{k}} \mathcal{B}_{\vec{m}}^{(j)} .
$$

So in what follows let us assume that all the centers of the balls are in $\mathrm{Q}^{0}$ and that $\sup _{\lambda \in L} r_{\lambda}=1$ by normalization.

First take a ball $B\left(x_{\lambda_{1}}, r_{\lambda_{1}}\right)$ arbitrarily from the family $\left\{B\left(x_{\lambda}, r_{\lambda}\right)\right\}_{\lambda \in L}$.

The assumption $1 / \sqrt{k}<\inf _{\lambda \in L} r_{\lambda}$ ensures that the radius of the ball is between $1 / \sqrt{k}$ and 1 . Thus the ball $B\left(x_{\lambda_{1}}, k r_{\lambda_{1}}\right)$ contains all the ball $B\left(x_{\lambda}, r_{\lambda}\right)$ such that $d\left(x_{\lambda}, x_{\lambda_{1}}\right)$ is less than $\sqrt{k}-1$.

Next take a ball $B\left(x_{\lambda_{2}}, r_{\lambda_{2}}\right)$ such that $d\left(x_{\lambda_{2}}, x_{\lambda_{1}}\right) \geq \sqrt{k}-1$. We may choose it arbitrarily as long as this condition is satisfied. As in the proceeding paragraph, the ball $B\left(x_{\lambda_{2}}, k r_{\lambda_{2}}\right)$ contains all the ball $B\left(x_{\lambda}, r_{\lambda}\right)$ such that $d\left(x_{\lambda}, x_{\lambda_{2}}\right)$ is less than $\sqrt{k}-1$.

In this way we repeatedly take a ball $B\left(x_{\lambda_{p}}, r_{\lambda_{p}}\right)$ such that $d\left(x_{\lambda_{p}}, x_{\lambda_{j}}\right)$ $\geq \sqrt{k}-1$ for all $j=1,2, \ldots, p-1$. This procedure will be stopped at $q$ th step when we obtain $\bigcup_{\lambda \in L} B\left(x_{\lambda}, r_{\lambda}\right) \subset \bigcup_{p=1}^{q} B\left(x_{\lambda_{p}}, k r_{\lambda_{p}}\right)$.

In fact this procedure stops in finite times: Precisely speaking, $q$ appearing in the last part is bounded by the constant that depends only on 
$k>1$ and $d$. Let us show this. Since all the radius of the ball is at most 1 , all the ball is contained in $[-1,2]^{d}$. By the construction of $\left\{x_{\lambda_{a}}\right\}$, we have $d\left(x_{\lambda_{a}}, x_{\lambda_{b}}\right) \geq \sqrt{k}-1$ for all $a<b \leq q$. Thus we have $q$ disjoint balls whose radii are more than $(\sqrt{k}-1) / 2$. Precisely speaking, $\left\{B\left(x_{j},(\sqrt{k}-\right.\right.$ $1) / 2)\}_{j=1, \ldots, q}$ is disjoint. And we have $B\left(x_{j},(\sqrt{k}-1) / 2\right)$ is contained in $[-1,2]^{d}$ for all $j=1, \ldots, q$. Hence we have $q((\sqrt{k}-1) / 2)^{d} V \leq 3^{d}$, where $V$ is volume of a unit ball. Thus $q$ is bounded by the quantity which depends only on $k>1$ and $d$. We put this bound $N$. If $q$ is less than $N$, we formally define $L_{j}=\emptyset$ for $j>q$. Peacing together these observations we are done.

Next we prove Theorem 1.5, that is, we want to eliminate the assumption

$$
\sup _{\lambda \in L} r_{\lambda}<\sqrt{k} \inf _{\lambda \in L} r_{\lambda}<\infty .
$$

Proof (of Theorem 1.5). Again we may assume that $\sup _{\lambda \in L} r_{\lambda}=1$. First we take the subfamilies $\mathcal{B}_{j, p}$ inductively as follows ( $j$ runs through all the positive integers and $p$ through $[1, N]$, where $N$ is a number obtained in the Lemma 3.1):

First we define $X_{1}$ as

$$
X_{1}=\left\{B\left(x_{\lambda}, r_{\lambda}\right) \mid r_{\lambda}>\frac{1}{\sqrt{k}}\right\} .
$$

Let $\mathcal{B}_{1, p}$ be families obtained from $X_{1}$, using the Lemma 3.1. Suppose we have obtained the families of the balls $\mathcal{B}_{l, p}$ with $l=1, \ldots, j, p=1, \ldots, N$ and that $X_{l}$ with $l=1, \ldots, j$ are defined as the subsets of $\left\{B\left(x_{\lambda}, r_{\lambda}\right)\right\}_{\lambda \in L}$. Then we define

$$
X_{j+1}=\left\{\begin{array}{l|l}
B=B\left(x_{\lambda}, r_{\lambda}\right) & \begin{array}{c}
\left(\frac{1}{\sqrt{k}}\right)^{j+1}<r_{\lambda} \leq\left(\frac{1}{\sqrt{k}}\right)^{j}, \\
B \not \subset \bigcup_{1 \leq p \leq N, 1 \leq l \leq j, B^{\prime} \in \mathcal{B}_{l, p}} k B^{\prime} .
\end{array}
\end{array}\right\},
$$

where $k B$ is an abbreviation of $B(x, k r)$ when $B=B(x, r)$. And we apply the Lemma 3.1 to this subset to obtain $\mathcal{B}_{j+1, p}$ with $p=1, \ldots, N$, which enjoy the following properties: 


$$
\begin{gathered}
\left\{\mathcal{B}_{j+1, p}\right\}_{1 \leq p \leq N} \text { are disjoint subfamilies and } \\
\bigcup_{B \in X_{j+1}} B \subset \bigcup_{p=1, \ldots, N} \bigcup_{B \in \mathcal{B}_{j+1, p}} k B .
\end{gathered}
$$

By the definition of $X_{j}$ we have,

(1) $B\left(x_{\lambda}, r_{\lambda}\right) \in X_{j}$ implies $r_{\lambda} \leq k^{-(j-1) / 2}$

(2) $B\left(x_{\lambda}, r_{\lambda}\right)$ is not contained in $\bigcup_{l=1}^{j-1} \bigcup_{p=1}^{N} \bigcup_{B \in \mathcal{B}_{l, p}} k B$.

Next we claim that there is an integer $N^{\prime}$, which depends only on $k$, that satisfies the following:

$$
\begin{aligned}
& \text { If }|j-l|>N^{\prime}, B^{\prime} \in \cup_{p} \mathcal{B}_{j, p} \text {, and } B \in \cup_{p} \mathcal{B}_{l, p}, \\
& \text { then we have } B \cap B^{\prime}=\emptyset \text {. }
\end{aligned}
$$

In fact suppose that $B \cap B^{\prime} \ni x$ and $l>j$. Then by property noted above, there exists $y \in B \backslash k B^{\prime}$. Let $c$ be the center of $B^{\prime}$. If $E$ is a subset of $\mathbf{R}^{d}, \operatorname{diam}(E)$ denotes the diameter of $E$. Under this notation and setting we have

$$
\begin{aligned}
& d(x, y) \leq \operatorname{diam}(B), d(c, y) \geq \frac{k}{2} \operatorname{diam}\left(B^{\prime}\right), \text { and } \\
& \qquad d(c, x) \leq \frac{1}{2} \operatorname{diam}\left(B^{\prime}\right) .
\end{aligned}
$$

Thus $((k-1) / 2) \operatorname{diam}\left(B^{\prime}\right)<\operatorname{diam}(B)$. By the construction of $X_{j}$, $\operatorname{diam}(B) \leq 2 /\left(\sqrt{k}^{l-1}\right)$ and $\operatorname{diam}\left(B^{\prime}\right) \geq 2 /\left(\sqrt{k}^{j}\right)$, hence we have $2 /\left(\sqrt{k}^{l-1}\right)$ $\geq(k-1) /\left(\sqrt{k}^{j}\right)$. Since $k>1$, this is possible only if the difference of $j$ and $l$ is small, that is,

$$
\frac{\log (2 \sqrt{k} /(k-1))}{\log \sqrt{k}}>l-j .
$$

Put $\mathcal{G}_{j, p}=\bigcup_{i: i \equiv j \bmod N^{\prime}} \mathcal{B}_{i, p}$. Then $\left\{\mathcal{G}_{j, p}\right\}_{j \leq N^{\prime}, p \leq N}$ does satisfy all the demands of the theorem.

Using this covering lemma we can prove Theorem 1.6.

Proof (of Theorem 1.6). We "cut off" the maximal function. Fix $R>0$ and define $M_{k, u c}^{R}$ as

$$
M_{k, u c}^{R} f(x)=\sup _{x \in B(z, r), R>r>0} \frac{1}{\mu(B(z, k r))} \int_{B(z, r)}|f(y)| d \mu(y) .
$$

(This notation is rather complicated but we want to emphasize that we are 
considering maximal operator with radii less than $R$.) Put

$$
E=\left\{x \in \mathbf{R}^{d} \mid M_{k, u c}^{R} f(x)>\lambda\right\} .
$$

By the definition of $E$, for all $x \in E$, there exist $y_{x} \in X$ and $0<r_{x}<R$ such that

$$
\mu\left(B\left(y_{x}, k r_{x}\right)\right) \leq \frac{1}{\lambda} \int_{B\left(y_{x}, r_{x}\right)}|f(x)| d \mu(x), x \in B\left(y_{x}, r_{x}\right) .
$$

We are restricting $r<R$ in the definition of $M_{k, u c}^{R}$ so we can use Theorem 1.5.

By the theorem, there exists $N_{k}$ and $x_{1}, x_{2}, \ldots \in\left\{y_{x} \mid x \in X\right\}$ such that

$$
1_{E}(x) \leq \sum_{j} 1_{B\left(x_{j}, k r_{x_{j}}\right)}(x) \text { and } \sum_{j} 1_{B\left(x_{j}, r_{x_{j}}\right)}(x) \leq N_{k}
$$

for all $x \in \mathbf{R}^{d}$.

Using this we obtain

$$
\begin{aligned}
& \mu(E) \leq \mu\left(\bigcup_{j} B\left(x_{j}, k r_{x_{j}}\right)\right) \\
\leq & \sum_{j} \mu\left(B\left(x_{j}, k r_{x_{j}}\right)\right) \leq \sum_{j} \frac{1}{\lambda} \int_{B\left(x_{j}, r_{x_{j}}\right)}|f(x)| d \mu(x) \\
= & \sum_{j} \frac{1}{\lambda} \int_{\mathbf{R}^{d}} 1_{B\left(x_{j}, r_{x_{j}}\right)}|f(x)| d \mu(x) \leq \frac{N_{k}}{\lambda} \int_{\mathbf{R}^{d}}|f(x)| d \mu(x) .
\end{aligned}
$$

Thus we have finished.

We consider another application of this covering lemma. This covering lemma allows us to obtain various estimates.

Theorem 3.1 We have the dual inequality

$$
\int_{\left\{M_{b, u c} f>\lambda\right\}}|g(x)| d \mu(x) \leq \frac{C_{a, b}}{\lambda} \int_{\mathbf{R}^{d}} M_{a, u c} g(x)|f(x)| d \mu(x) .
$$

if $b>a>1$, where $C_{a, b}$ is a positive constant depending on $a, b$ and $d$.

Proof. Fix $R>0$. We "cut off" the maximal function again. Put again

$$
M_{b, u c}^{R} f(x)=\sup _{x \in B(z, r), R>r>0} \frac{1}{\mu(B(z, b r))} \int_{B(z, r)}|f(y)| d \mu(y) .
$$


Fix $\lambda>0$. We set

$$
E_{b}=\left\{x \in \mathbf{R}^{d} \mid M_{b, u c}^{R} f(x)>\lambda\right\} .
$$

For all $x \in E_{b}$ by its definition there exists $r_{x}<R$ and $y_{x}$ such that

$$
\frac{1}{\mu\left(B\left(y_{x}, b r_{x}\right)\right)} \int_{B\left(y_{x}, r_{x}\right)}|f(z)| d \mu(z)>\lambda \text { and } x \in B\left(y_{x}, r_{x}\right) .
$$

Note that $\sup _{x \in E_{b}} r_{x}$ is at most $R$. So we can apply Theorem 1.5. Applying the theorem with $b / a>1$, we obtain a countable subset $A \subset E_{b}$ such that $\left\{B\left(y_{x}, r_{x}\right)\right\}_{x \in A}$ satisfies

$$
\bigcup_{x \in E_{b}} B\left(y_{x}, r_{x}\right) \subset \bigcup_{j} B\left(x_{j}, \frac{b}{a} r_{j}\right) \text { and } \sum_{j} 1_{B\left(x_{j}, r_{j}\right)} \leq C_{a, b} .
$$

Using these properties, we have

$$
\begin{aligned}
& \int_{E_{b}} g(x) d \mu(x) \leq \int_{\bigcup_{j} B\left(x_{j},(b / a) r_{j}\right)} g(x) d \mu(x) \\
\leq & \sum_{j} \frac{1}{\mu\left(B\left(x_{j}, b r_{j}\right)\right)} \int_{B\left(x_{j},(b / a) r_{j}\right)} g(x) d \mu(x) \times \mu\left(B\left(x_{j}, b r_{j}\right)\right) \\
\leq & \sum_{j}\left(\inf _{x \in B\left(x_{j}, r_{j}\right)} M_{a, u c}^{R} g(x)\right) \frac{1}{\lambda} \int_{B\left(x_{j}, r_{j}\right)}|f(y)| d \mu(y) \\
\leq & \frac{C_{a, b}}{\lambda} \int_{\mathbf{R}^{d}}|f(y)| M_{a, u c}^{R} g(y) d \mu(y) .
\end{aligned}
$$

Tending $R$ to $\infty$, we are done.

As a corollary we have Theorem 1.7 whose proof is obtained by changing the parameters in Theorem 1.3 suitably.

Acknowledgment The author is grateful of Prof. Yasuo Komori in Tokai University. Without his constant warm-hearted encouragement, the author could not write this paper.

I also express deep gratitude to Dr. Yutaka Terasawa, Dr. Xu Bin, and Prof. Hitoshi Arai in the University of Tokyo. They kindly pointed out many vital errors of the preprint paper. Finally I thank the referee for his kind advice and careful reading of this paper. 


\section{References}

[1] Carlsson H., A new proof of the Hardy-Littlewood maximal theorem. Bull. London Math. Soc. 16 (1984), 595-596.

[2] C. Evans L. and F. Gariepy R., Measure theory and fine properties of functions. C. Evans L. and F. Gariepy R. (Studies in advanced mathematics) CRC Press, 1999.

[ 3 ] Cuerva J-G. and Martell J.M., Weighted inequalities and vector-valued CarderónZygmund operators on nonhomogeneous spaces. Publ. Mat. 44 (2) (2000), 613-640

[4] Cuerva J-G. and Martell J.M., On the existence of principal values for the Cauchy integral on weighted Lebesgue spaces for non-doubling measures. J. Fourier Anal. Appl. 7 (5) (2001), 469-487.

[ 5 ] Guzman D., Real Variable Methods in Fourier Analysis. North-Holland Mathematical Studies, 46, Notas de Matematica(75) (1981).

[6] Journé J., Calderón-Zygmund operators, pseudo differential operators and the Cauchy integral of Calderón. Lecture Notes in Math., vol. 994, Springer-Verlag, Berlin, 1983.

[ 7 ] Mattila P., Geometry of sets and measures in Euclidean spaces. Fractals and rectifiability, 44, Cambridge Studies in Advanced Mathematics, 1995

[8] Nazarov F. Treil S. and Volberg A., Weak type estimates and Cotlar inequalities for Calderón-Zygmund operators on nonhomogeneous spaces. Internat. Math. Res. Notices, no. 9 (1998), 463-487.

[9] Terasawa Y., Outer measure and Weak- $(1,1)$ estimates of the modified HardyLittlewood maximal operators. (2004), in preparation.

[10] Tolsa X., BMO, $H^{1}$, and Carderón-Zygmund operators for non doubling measures. Math. Ann. 319 (2001), 89-149.

Graduate School of Mathematical Sciences The University of Tokyo

3-8-1 Komaba, Meguro-ku

Tokyo 153-8914, Japan

E-mail: yosihiro@ms.u-tokyo.ac.jp 